\title{
Stable, Sensitive, Fluorescence-Based Method for Detecting cAMP
}

BioTechniques 33:691-694 (September 2002)

\author{
Jayne Hesley, Janet Daijo, \\ and Anne T. Ferguson \\ Molecular Devices, \\ Sunnyvale, CA, USA
}

\footnotetext{
ABSTRACT

cAMP is a universal secondary messenger that connects changes in the extracellular environment, as detected by cell surface receptors, to transcriptional changes in the nucleus. Since cAMP-mediated signal transduction plays a role in critical cell functions and human diseases, monitoring its activity can aid in understanding these responses and the process of drug discovery. This report examines the performance of a fluorescence-based competitive immunoassay in 384-well microplate format. Using purified cAMP as a competitor, the estimated detection limit was determined to be $0.1 \mathrm{nM}$ and $Z^{\prime}$-factor was greater than 0.83 , which indicates that the assay is of high quality and one of the most sensitive assays currently on the market. Of note, the results obtained were similar whether the reaction was allowed to proceed for $10 \mathrm{~min}$ or up to $60 \mathrm{~min}$. Next, HEK 293 cells were treated with the promiscuous adenylate cyclase activator, forskolin, and the $\beta$-adrenoceptor agonist,
}

isoproterenol. The resultant average $E C_{50}$ values were $11 \mu \mathrm{M}$ and $123 \mathrm{nM}$, respective$l y$, which correspond to those found in the literature. Together, these results demonstrate that this assay is a fast, accurate, nonradioactive method that is ideal for highthroughput screening.

\section{INTRODUCTION}

cAMP is involved in intra- and intercellular signaling in organisms as diverse as bacteria, slime mold, fruit flies, and humans $(4,5,9)$. cAMP is produced from ATP by the enzyme adenylate cyclase and is hydrolyzed by cAMP phosphodiesterases. Regulation of these enzymes allows for tight control over the levels of cAMP inside the cell. In mammals, extracellular ligands, such as peptide hormones and neurotransmitters, interact with transmembrane proteins called G-coupled receptors. Ligand binding leads to a conformational change in the receptor that allows its association with a GTP-binding regulatory protein (G-protein). G-coupled receptors activate adenylate cyclase when bound to a stimulatory form of G-protein, $\mathrm{G}_{\mathrm{s}}$, and inhibit the enzyme when bound to an inhibitory form of G-pro- tein, $\mathrm{G}_{\mathrm{i}}$. Activation of $\mathrm{G}_{\mathrm{s}}$-coupled receptors results in increased production of cAMP, which in turn activates cAMPdependent protein kinase (PKA). This enzyme phosphorylates a variety of proteins, which eventually leads to the regulation of transcription factor activity and effects on gene expression. Targets for PKA include other enzymes, ion channels, and transcriptional regulators. In addition, cAMP plays a direct role in transcription by binding and augmenting the activity of the transcription factor, cAMP-responsive element-binding protein (CREB). This protein plays a role in modulating the expression of cAMP-inducible genes (6). Further complicating this pathway is the role that cAMP plays in directly regulating the activity of cation channels (10).

Depending on the cell type, this signal transduction pathway may be responsible for neural and immune responses, metabolism, mitogenesis oocyte maturation, and many other critical functions $(2,5,7,8,10,11)$. Therefore, an accurate method for quantitative measurement of intracellular cAMP is critical for basic research and drug discovery purposes. The new fluorescence intensity-based assay described here is unique because readings 
Table 1. Instrument Settings for the Analyst AD

\begin{tabular}{|ll|}
\hline Parameter & Settings \\
\hline Mode & $\begin{array}{l}\text { Fluorescence } \\
\text { intensity }\end{array}$ \\
Emission filters & Rhodamine \\
& $530-25 \mathrm{~nm}$ \\
Dichroic mirror & $50 / 50$ Beam- \\
& splitter \\
Z-height & 3 mm \\
Attenuator & Medium \\
Integration time & $50000 \mu$ s \\
Lamp & Continuous \\
Readings/well & One \\
PMT Setup & Smart Read + \\
& (sensitivity = 2) \\
Units & cps \\
\hline
\end{tabular}

can be taken in as little as 10 min or as long as $24 \mathrm{~h}$ after substrate addition, since no termination step is needed. Also, the overall time to process the assay is approximately $3 \mathrm{~h}$, and it includes only one wash step. Most importantly, the assay is one of the most sensitive assays currently available on the market. Here we illustrate these advantages and the high quality of the assay.

\section{MATERIALS AND METHODS}

\section{Cells}

HEK 293 cells (human embryonic kidney cells that stably overexpress the Adenovirus E1A protein) were grown in DMEM with $10 \% \mathrm{FBS}$ at $37^{\circ} \mathrm{C}$ in $5 \% \mathrm{CO}_{2}$.

\section{Reagents}

The reagents used were CatchPoint $^{\mathrm{TM}}$ cAMP fluorescent assay kit that includes solid black, 384-well microplates pre-coated with goat anti-rabbit antibody (Molecular Devices, Sunnyvale, CA, USA), DMSO, low water content (Sigma, St. Louis, MO, USA), Krebs-Ringer Bicarbonate Buffer (KRBG; Sigma), sodium bicarbonate (Sigma), cAMP agonists including forskolin (Sigma) and isoproterenol (Sigma), phosphodiesterase inhibitor, 3-isobutyl-1-methylxanthine (IBMX; Sigma), and $30 \%$ hydrogen peroxide solution (Sigma).

\section{Cell Preparation}

HEK 293 cells were grown to approximately $80 \%$ confluence at $37^{\circ} \mathrm{C}$. Cells were detached using $0.526 \mathrm{mM}$ EDTA in PBS and washed once with KRBG. Cells were resuspended at a concentration of $1 \times 10^{6}$ cells $/ \mathrm{mL}$ in stimulation buffer (KRBG buffer that contains $0.75 \mathrm{mM}$ IBMX). Next, cells were seeded in a volume of $7.5 \mu \mathrm{L}$ at a concentration of $0.75 \times 10^{4}$ cells/well of a 384-well microplate. The microplate was incubated for $10 \mathrm{~min}$ at room temperature.

\section{Cell Stimulation}

To stimulate cells to produce cAMP, $15-\mu \mathrm{L}$ doses of activator and agonist were diluted in PBS (forskolin and isoproterenol; Sigma); for unstimulated cell samples, PBS alone was added. The range of agonist final concentrations used was $0-1 \mathrm{mM}$. In addition, $15-\mu \mathrm{L}$ samples of cAMP calibrator at final concentrations ranging from 0 to $3333 \mathrm{nM}$ were added to the wells to generate a calibration curve. Each of the above conditions was performed in replicates of eight. The microplate was gently mixed and incubated at room temperature for $15 \mathrm{~min}$. To lyse the cells, $7.5 \mu \mathrm{L}$ cell lysis buffer (supplied with kit) were added to each well, and the plate was agitated for $10 \mathrm{~min}$ at room temperature using a plate shaker. The final volume in each well was $30 \mu \mathrm{L}$.

\section{Immunoassay}

The immunoassay procedure was started by transferring $20 \mu \mathrm{L}$ each sample to the 384-well solid black assay microplate (supplied with kit). The samples were then treated with $20 \mu \mathrm{L}$ rabbit anti-cAMP antibody (supplied with the kit), and the microplate was gently agitated on a plate shaker for 5 min to ensure proper mixing. Next, $20 \mu \mathrm{L}$ HRPcAMP conjugate (supplied with kit) were added to all wells, and the plate was agitated and incubated for $2 \mathrm{~h}$ at room temperature. The assay microplate was washed four times with 80 $\mu \mathrm{L} /$ well of wash buffer (supplied with kit). Then, $50 \mu \mathrm{L}$ Stoplight Red solution (supplied with kit) was added to every well. The microplate was covered to protect it from light and incubated for up to $1 \mathrm{~h}$ at room temperature. The resulting fluorescence intensity was read at $10 \mathrm{~min}$ and $60 \mathrm{~min}$ using a Gemini XS and Analyst ${ }^{\mathrm{TM}}$ AD (both from Molecular Devices). The instrument settings used for the Gemini XS were excitation wavelength of $530 \mathrm{~nm}$, emission wavelength of $590 \mathrm{~nm}$, emission cut-off filter of $570 \mathrm{~nm}$, and six readings/well with the photomultiplier tube set to automatic. Table 1 shows the instrument settings used for the Analyst AD.

\section{RESULTS}

\section{Assay Performance}

Analysis of the data obtained for samples containing cAMP calibrator resulted in the plots shown in Figure 1. These data were obtained after $10 \mathrm{~min}$ and $60 \mathrm{~min}$ incubation with Stoplight Red substrate. The coefficient $\mathrm{C}$ is the $\mathrm{X}$-value corresponding to the $\mathrm{Y}$-value that is the midpoint between the low and high values on the asymptote of the curve fit to the 4-parameter equation: $\mathrm{Y}=(\mathrm{A}-\mathrm{D}) /\left[1+(\mathrm{X} / \mathrm{C})^{\mathrm{B}}\right]+\mathrm{D}$. This equation is shown below the graphs in all of the figures. The value for coefficient $\mathrm{C}$ is equivalent to the $\mathrm{EC}_{50}$ value. In Figure 1 , the value for the coefficient $\mathrm{C}$ $\left(\mathrm{EC}_{50}\right.$ value) is $2.4 \mathrm{nM}$ for $10 \mathrm{~min}$ (Figure 1A) and $3.7 \mathrm{nM}$ for $60 \mathrm{~min}$ (Figure 1B). The $\mathrm{EC}_{50}$ values read at $10 \mathrm{~min}$ and 60 min were similar, which demonstrated the relative stability of the fluorescent signal over time. The signal is also stable for $24 \mathrm{~h}$ (data not shown). The average $\mathrm{EC}_{50}$ value obtained when the assay was performed on different days was $3.55 \pm 0.2 \mathrm{nM}$. This value is in agreement with the average $\mathrm{EC}_{50}$ value of $3.4 \pm 0.6 \mathrm{nM}$, which was obtained using the Analyst AD.

For the experiments shown in Figure 1 , the signal-to-noise ratio (no cAMP calibrator) was 240 , and the limit of detection (LOD, defined as three standard deviations difference from the zero cAMP calibrator control) was $0.1 \mathrm{nM}$ 
cAMP for both fluorescence microplate readers. The optimal performance range of the assay falls between 0.14 and 33 $\mathrm{nM}$. These results are in accordance with those obtained using the Analyst $\mathrm{AD}(0.1-80 \mathrm{nM}$, data not shown). In addition, the $Z^{\prime}$-factor for the assay was calculated. The concentration of cAMP calibrator that achieved maximal inhibition ( $33 \mathrm{nM}$ ) was used as the positive control, and the concentration of cAMP calibrator that achieved the minimal inhibition $(0.14 \mathrm{nM})$ was used as the negative control. A value between 0.5 and 1.0 indicates a high-quality, robust assay appropriate for screening purposes (12).
The $Z^{\prime}$-factors for the assay read on the Gemini XS and Analyst AD were 0.84 and 0.90 , respectively, when using eight replicates. In a separate experiment, the $\mathrm{Z}^{\prime}$-factor was calculated using 64 replicates of $33 \mathrm{nM}$ calibrator and 64 replicates of $0.14 \mathrm{nM}$ calibrator and read using the Analyst $\mathrm{AD}$. The resulting $\mathrm{Z}^{\prime}$-factor value was 0.89 .

\section{Response of HEK 293 Cells to Forskolin and Isoproterenol}

Next, the response of HEK 293 cells to forskolin and isoproterenol was examined. Forskolin is a natural diter- pene, which is a promiscuous activator of all adenylate cyclase isoforms, while isoproterenol is a $\beta$-adrenoceptor agonist $(1,3)$. HEK 293 cells are a suitable model system with which to study the regulation of cAMP because of their low endogenous level of adenylate cyclase activity (3). The results of the experiments are shown in Figures 2 and 3 . The $\mathrm{EC}_{50}$ values obtained for forskolin were $12.7 \mathrm{nM}$ and $9.3 \mathrm{nM}$ using Gemini $\mathrm{XS}$ and Analyst AD, respectively (Figure 2). These results are in accordance with values obtained using other products $\left([\mathrm{FP}]^{2 \mathrm{TM}}\right.$ kit from $\mathrm{NEN}^{\circledR}$ Life Science Products; Perkin Elmer Life
A

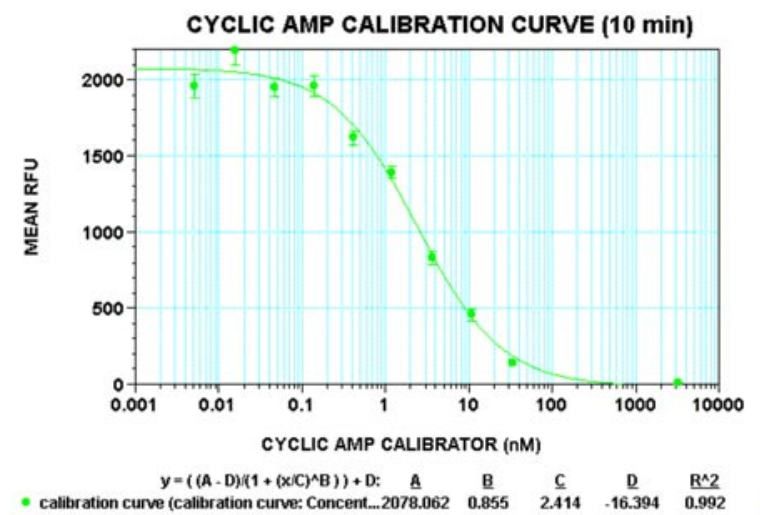

B

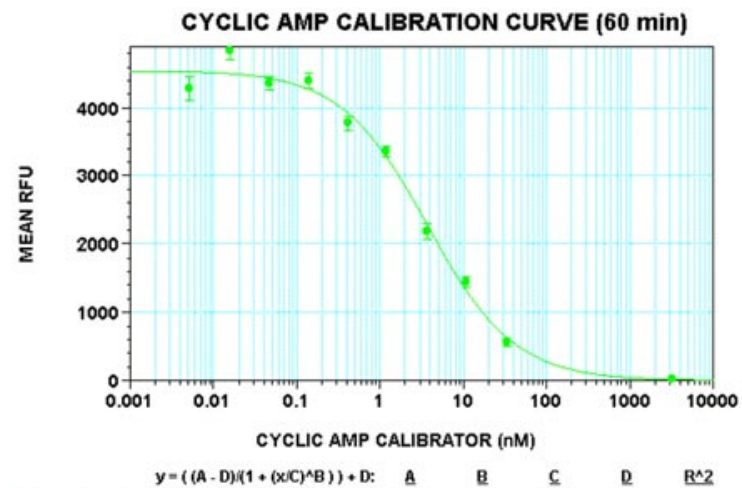

Figure 1. Dose response curves for cAMP calibrator using Gemini XS. Dose responses are shown for (A) $10 \mathrm{~min}$ and (B) $60 \mathrm{~min}$ after the addition of Stoplight Red substrate. Each point on the dose response curves represents the average of eight replicate samples. Error bars denote the standard deviation from the mean. $\mathrm{R}^{2}$ value is 0.99 for a 4-parameter curve fit.

A

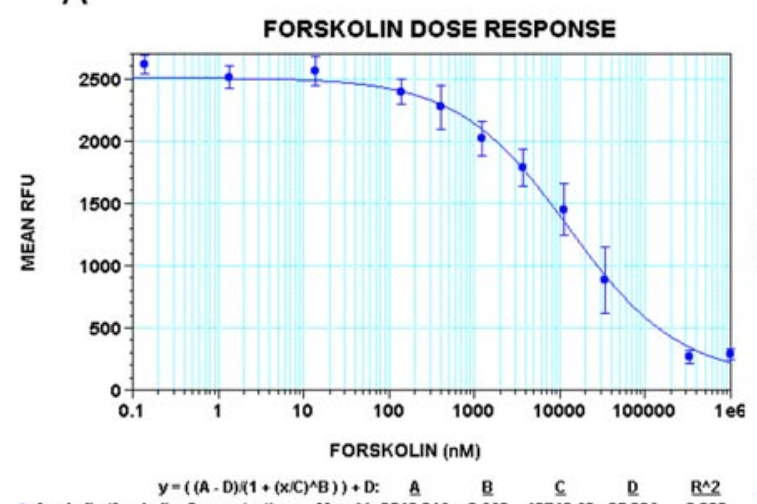

B

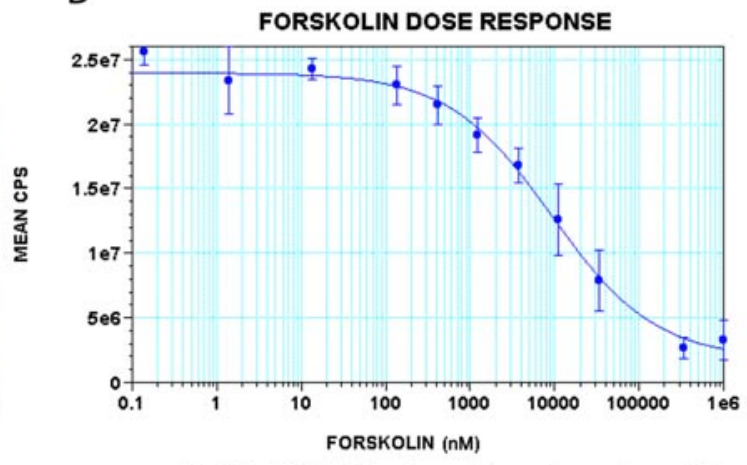

$y=\left((A-D)\left(1+(x / C)^{\wedge} B\right)\right)+D: \quad A \quad$ B $\quad$ C $\quad$ D $\quad B^{\wedge} 2$

Figure 2. Dose response curves for forskolin-treated HEK 293 cells. Each point on dose response curves represents average RFU of eight replicate samples for one concentration of the activator. The plate was incubated with Stoplight Red substrate for $60 \mathrm{~min}$ before reading this data set. Error bars denote the standard deviation from the mean. $\mathrm{R}^{2}$ value for the experiment was 0.99 using a 4-parameter curve fit. Curve shown in panel A was obtained using Gemini XS, and the curve shown in panel B was obtained using Analyst AD. The $\mathrm{EC}_{50}$ values were (A) $12.7 \mathrm{nM}$ and (B) $9.3 \mathrm{nM}$ for Gemini XS and Analyst $\mathrm{AD}$, respectively. 
A

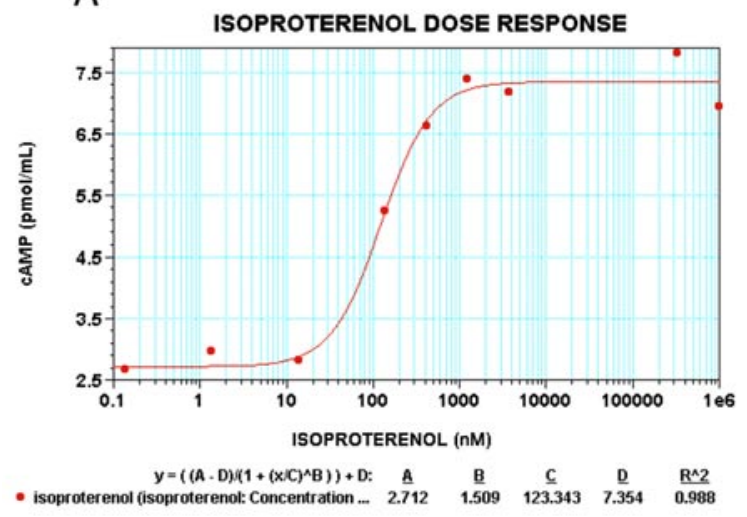

B

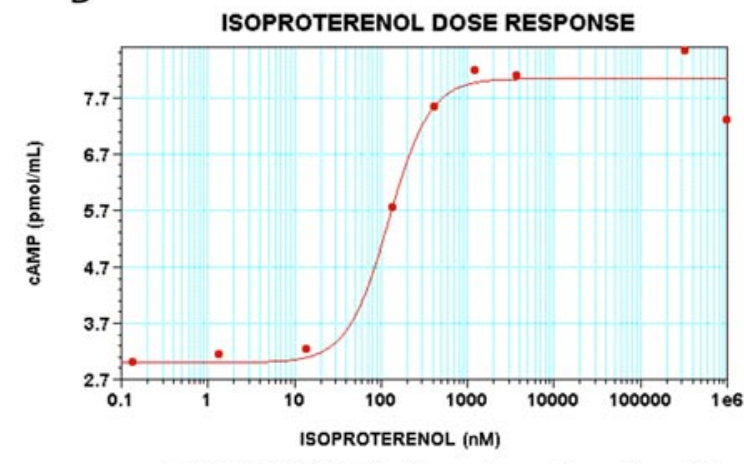

$\begin{array}{cccccc}\left.y=\left((A-D)(1+(X) C)^{\wedge} B\right)\right)+D: & B & B & C & D & \frac{B}{A} 2 \\ \text { (isoproterenot: Concentration } \ldots & 2.995 & 1.812 & 122.924 & 8.046 & 0.983\end{array}$

Figure 3. Dose response curves for isoproterenol-treated HEK 293 cells. Each point on the dose response curves represents the average cAMP of eight replicate samples for one concentration of the agonist. The $\mathrm{R}^{2}$ value for both experiments was 0.98 for a 4-parameter curve fit. The dose response curves shown were obtained after 60 min incubation of samples with Stoplight Red substrate. The curve shown in panel A was obtained using Gemini XS, and the curve shown in panel B was obtained using Analyst AD. The cAMP values shown on the Y-axis were obtained by interpolation of the original data. The $\mathrm{EC}_{50}$ values were (A) $123 \mathrm{nM}$ and (B) $123 \mathrm{nM}$ for Gemini XS and Analyst AD, respectively.

Sciences, Gaithersburg, MD, USA).

The $\mathrm{EC}_{50}$ values obtained for isoproterenol were $123 \mathrm{nM}$ using both the Gemini XS and Analyst AD, respectively (Figure 3 ). The cAMP values shown on the Y-axis were obtained by interpolation of the original data. The graphs in Figure 3 were shown to demonstrate the ability of the software supplied with both fluorescence microplate readers to convert relative fluorescence unit (RFU) to cAMP values. The $\mathrm{EC}_{50}$ values for isoproterenol are in accordance with values obtained using another cell line (1).

\section{DISCUSSION}

The CatchPoint cAMP fluorescent assay is very sensitive and has an LOD of $0.1 \mathrm{nM}$. In comparison to the Molecular Devices fluorescence polarization method for measuring cAMP (1.25 $\mathrm{nM})$, Catchpoint is approximately 10fold more sensitive. The assay kit has a short post-substrate addition incubation time required to reach a stable signal (10 $\min$ to $24 \mathrm{~h}$ ). This makes the kit ideal for using a stacker and robotic system to transfer the microplates to a fluorescence microplate reader that may be used in screening laboratories. This point is confirmed by comparison of the results after $10 \mathrm{~min}$ and $60 \mathrm{~min}$ incubation with Stoplight Red substrate. The $\mathrm{LOD}$ and $\mathrm{EC}_{50}$ values for the cAMP calibrator were very similar at the two time points. Furthermore, this fluorescence assay provides a high-quality screening assay, as demonstrated by the $\mathrm{Z}^{\prime}$-factor and similar results obtained on different days and with different fluorescence microplate readers. In conclusion, CatchPoint cAMP fluorescent assay allows simple, convenient, and highly sensitive quantitative measurement of intracellular cAMP.

\section{REFERENCES}

1.Cary, D.A. and F.A.O. Mendelsohn. 1987. Effect of forskolin, isoproterenol and IBMX on angiotension converting enzyme and cyclic AMP production by cultured bovine endothelial cells. Mol. Cell. Endocrinol. 53:103-109.

2.Etgen, A.M., M.A. Ansonoff, and A. Quesada. 2001. Mechanisms of ovarian steroid regulation of norepinephrine receptor-mediated signal transduction in the hypothalamus: implications for female reproductive physiology. Horm. Behav. 40:169-177.

3.Hanoune, J., Y. Pouille, E. Tzavara, T. Shen, L. Lipskaya, N. Miyamoto, Y. Suzuki, and N. Defer. 1997. Adenylyl cyclases: structure, regulation and function in an enzyme superfamily. Mol. Cell. Endocrinol. 128:179-194.

4.Maree, A.F. and P. Hogeweg. 2001. How amoeboids self-organize into a fruiting body: multicellular coordination in Dictyostelium discoideum. Proc. Natl. Acad. Sci. USA 98:3879-3883.

5.Martin, F., M.J. Charro, and E. Alcorta. 2001. Mutations affecting the cAMP transduction pathway modify olfaction in Drosophila. J. Comp. Physiol. 187:359-370.

6.Mayr, B.M., G. Canettieri, and M.R. Montminy. 2001. Distinct effects of cAMP and mi- togenic signals on CREB-binding recruitment impart specificity to target gene activation via CREB. Proc. Natl. Acad. Sci. USA 98:1093610941.

7.Monneret, G., S. Gravel, M. Diamond, J. Rokach, and W.S. Powell. 2001. Prostaglandin $\mathrm{D}(2)$ is a potent chemoattractant for human eosinophils that acts via a novel DP receptor. Blood 98:1942-1948.

8.Moutinho, A., P.J. Hussey, A.J. Trewavas, and R. Malho. 2001. cAMP acts as a secondary messenger in pollen tube growth and reorientation. Proc. Natl. Acad. Sci. USA 98:10481-10486.

9.Vossen, K.M., R. Wolz, M.A. Daugherty, and M.G. Fried. 1997. Role of macromolecular hydration in the binding of the Escherichia coli cyclic AMP receptor to DNA. Biochemistry 36:11640-11647.

10.Weiner, R.I. and A. Charles. 2001. Regulation of gonadotropin-releasing hormone release by cyclic AMP signalling pathways. Growth Horm. IGF Res. 11:S9-S15.

11.Zalatan, F., J.A. Krause, and D.E. Blask. 2001. Inhibition of isoproterenol-induced lipolysis in rat inguinal adipocytes in vitro by physiological melatonin via a receptor-mediated mechanism. Endocrinology 142:3783-3790.

12.Zhang, J.-H., T.D.Y. Chung, and K.R. Oldenburg. 1999. A simple, statistical parameter for use in evaluation and validation of highthroughput screening assays. J. Biomol. Screen. 4:67-73.

\section{Address correspondence to:}

Dr. Anne T. Ferguson

Molecular Devices

1311 Orleans Drive

Sunnyvale, CA 94089, USA

e-mail: anne_ferguson@moldev.com 